\title{
The Role of a Boost Radiation Dose in Patients with Negative Re-Excision Findings
}

\author{
Melanie C. Smitt and Kathleen C. Horst ${ }^{*}$ \\ Department of Radiation Oncology, Stanford University, 875 Blake Wilbur Dr, Stanford, CA 94305, USA
}

\begin{abstract}
The aim of this study is to evaluate whether patients who have no residual tumor at the time of a re-excision lumpectomy benefit from the addition of a radiation boost dose. The records of 535 patients treated with breastconserving therapy (BCT) for Stage I-II breast cancer were reviewed. The actuarial risk of local recurrence (LR) was determined for the 262 patients in this cohort who underwent re-excision lumpectomy. There was no significant difference in the 10-year freedom from local recurrence (FFLR) for patients with negative re-excisions (96\%) compared to patients with residual tumor that was adequately excised (92\%). Patients with residual tumor at or close to the final margins, however, had a significantly lower FFLR $(80 \%$; $\mathrm{p}=.01)$. The FFLR for patients with negative re-excisions was $97 \%$ for patients not receiving a boost $(n=94)$ and $95 \%$ for patients who received a boost $(n=74)(p=n s)$. For patients $<50$, FFLR was $96 \%$ with a boost and $87 \%$ without a boost $(\mathrm{p}=\mathrm{ns})$. Extensive intraductal component was the only significant predictor of LR among patients with negative re-excisions $(\mathrm{p}=.02)$. The presence of residual disease in the re-excision specimen did not predict for LR as long as the final margins were negative. Among patients with negative re-excisions, the boost did not significantly improve local control. There was a trend for better outcomes, however, in younger patients treated with a boost even in the setting of a negative re-excision. Due to the limitations of the retrospective nature of this study, it will be important to address this question in a prospective fashion before making any definitive conclusions or changes in current clinical practice.
\end{abstract}

Keywords: Breast, radiotherapy, boost, re-excision.

\section{INTRODUCTION}

Several prospective randomized trials have demonstrated that breast-conserving surgery (BCS) followed by wholebreast radiotherapy (WBRT) is an appropriate treatment option for patients with Stage I and II breast cancer [1]. In addition to preventing local recurrences, WBRT after BCS has been shown to improve breast cancer specific mortality and overall survival [2]. After three randomized trials demonstrated that a "boost" dose to the lumpectomy cavity further reduces the risk of local recurrence [3-5], radiotherapy treatment plans after BCS for invasive carcinoma often consist of WBRT followed by a 10-16 Gy boost to the lumpectomy cavity. The added cost of a boost dose [6] and its effects on cosmesis [7], however, have led some to propose that select patients, such as those with negative re-excisions, may have acceptable local control rates with WBRT alone and could therefore avoid the boost treatment [8]. Whether these patients who have no residual disease at the time of re-excision lumpectomy have improved local control with the addition of a boost radiation dose is unclear.

Furthermore, a recent study found that, among patients who underwent whole breast radiation plus a routine boost, those with negative re-excisions had a significantly lower risk of local recurrence at 10 years compared to patients who

*Address correspondence to this author at the Department of Radiation Oncology, Stanford University, 875 Blake Wilbur Dr, Stanford, CA 94305, USA; Tel: 650-736-7715; Fax: 650-725-8231;

E-mails:kateh@stanford.edu; horstkathleen@hotmail.com had residual tumor in the re-excision specimen $(5 \%$ versus 9\%) [9]. These results suggest that those patients who achieve a wider margin, defined by a negative re-excision, have a more favorable outcome with the boost dose compared to those with residual disease and smaller margins.

The goal of the current study is to determine whether patients with no residual tumor identified in the re-excision lumpectomy specimen have better outcomes compared to those with residual disease, and whether a boost radiation dose after WBRT further improves local control in those patients with a negative re-excision.

\section{MATERIALS AND METHODS}

The records of 535 patients treated with breastconserving surgery and radiotherapy at Stanford University or the Washington-Stanford Radiation Oncology Center during the years 1972-1996 for Stage I or II breast cancer were reviewed with IRB approval. Clinical and pathologic data were recorded as previously described [10]. Margin status was classified on the initial and re-excision specimens as positive when invasive or in-situ disease was seen at an inked surgical margin, close when tumor cells were $\leq 2 \mathrm{~mm}$ from the ink, negative if tumor was $>2 \mathrm{~mm}$ from the inked margin, or indeterminate when the specimen was removed in pieces without orientation or not inked. Pathologic specimens were routinely reviewed at Stanford prior to radiotherapy treatment.

Radiation treatment policies varied over the years of the study. Most commonly, patients received 50.4 Gy prescribed to the isocenter in 1.8 Gy fractions followed by a $10 \mathrm{~Gy}$ electron boost prescribed at dmax to the tumor bed using 
ultrasound guidance. In earlier years patients could have received interstitial Iridium 192 implant boosts or reduced photon fields. Overall $65 \%$ of patients received a total tumor bed dose of $\geq 60 \mathrm{~Gy}$. Forty-seven percent of patients with negative margins did not receive a dose of $\geq 60 \mathrm{~Gy}$, according to individual physician preference and/or availability of electron beam equipment. Approximately $30 \%$ of patients received chemotherapy. Most commonly, patients received CMF concurrent with radiation $(n=112)$. The mean follow-up for surviving patients is eight years.

The actuarial probability of freedom from local recurrence as a first failure was calculated using the KaplanMeier method. Analysis of potential prognostic factors was performed using Cox regression analysis (SPSS, Inc.). Factors were included in the forward conditional Cox multivariate analysis if the univariate $p$ value was $\leq .05$. Factors included in the regression analysis for local recurrence among patients with negative re-excisions were: patient age $(<>>50)$, pathologic tumor size, pathologic nodal status (N0/N1), EIC (present/absent), estrogen receptor status (positive/negative), use of systemic adjuvant therapy (yes/no), and total radiation dose ( $<60 \mathrm{~Gy}, \geq 60 \mathrm{~Gy}$ ).

\section{RESULTS}

The overall outcome of the 535 patients treated with breast-conservation therapy has been previously reported by our group [10,11]. Of these patients, 262 underwent reexcision lumpectomy for close, positive, or indeterminate margins. On final pathology of the re-excision specimens, 168 patients (64\%) had no residual tumor, 91 (35\%) had either residual invasive or in-situ carcinoma, and results were unknown for 3 patients (1\%). Patient characteristics for the entire cohort of 535 patients and the 168 patients with negative re-excision findings are given in Table $\mathbf{1}$.

For all patients who achieved negative final margins $(\mathrm{n}=342$, including patients with negative margins after the initial surgery as well as after re-excision lumpectomy), the actuarial freedom from local recurrence at ten years (FFLR) is $94 \%$. Among patients who underwent re-excision, the presence or absence of residual disease in the specimen was not a predictor of outcome, as long as final margins were negative $(>2 \mathrm{~mm})$. The FFLR for patients with no residual tumor was $96 \%$ versus $92 \%$ for patients with residual tumor that was adequately excised $(\mathrm{p}=\mathrm{ns})$. Patients who underwent re-excision and still had close, positive, or unknown margins, however, had a FFLR of $80 \%(\mathrm{p}=.01$ versus re-excision with negative margins). Recurrence rates by re-excision findings are given in Table 2 .

Among the 168 patients with negative re-excision findings, there was no significant difference in the FFLR for patients in whom the boost radiation dose was omitted (97\%; $\mathrm{n}=94)$ compared to those who received a boost $(95 \% ; \mathrm{n}=74)$. Patients with a negative re-excision who were less than 50 years of age, however, had an $87 \%$ FFLR without boost radiation and $96 \%$ FFLR with boost radiation $(\mathrm{p}=\mathrm{ns})$. The recurrence rates by age and use of boost for patients with no residual tumor are shown in Table 3 . Of the five patients with negative re-excision findings who had a local recurrence, 2 were considered "true" tumor bed recurrences, and 3 could not be classified based on available information.
Table 1. Patient Characteristics

\begin{tabular}{|c|c|c|c|}
\hline & & $\begin{array}{l}535 \text { Treated } \\
\text { Patients }\end{array}$ & $\begin{array}{l}168 \text { patients with } \\
\text { Negative Re-Excision }\end{array}$ \\
\hline \multicolumn{4}{|l|}{ Age } \\
\hline & $<50$ & 197 & 56 \\
\hline & $\geq 50$ & 338 & 112 \\
\hline \multicolumn{4}{|c|}{ Pathologic T stage } \\
\hline & $\mathrm{T} 1$ & 399 & 138 \\
\hline & $\mathrm{T} 2$ & 108 & 25 \\
\hline & Tx & 28 & 5 \\
\hline \multicolumn{4}{|c|}{ Pathologic N stage } \\
\hline & No & 350 & 130 \\
\hline & N1 & 117 & 28 \\
\hline & NX & 68 & 10 \\
\hline \multicolumn{4}{|c|}{ Re-excision } \\
\hline & Yes & 262 & \\
\hline & -- No residual & 168 & \\
\hline & -- Residual & 91 & \\
\hline & -- Unknown & 3 & \\
\hline & No & 273 & \\
\hline \multicolumn{4}{|l|}{ EIC } \\
\hline & Present & 60 & 14 \\
\hline & Absent & 308 & 93 \\
\hline & Not available & 167 & 61 \\
\hline \multicolumn{4}{|c|}{ Total tumor bed radiation dose } \\
\hline & $<60 \mathrm{~Gy}$ & 189 & 94 \\
\hline \multirow[t]{2}{*}{$\geq 60$ Gy } & 345 & 74 & \\
\hline & Unknown & 1 & \\
\hline \multicolumn{4}{|c|}{ Systemic therapy } \\
\hline & Chemo & 153 & 44 \\
\hline & Tamoxifen & 172 & 58 \\
\hline
\end{tabular}

Table 2. 10 Year Freedom from Local Recurrence Rates by Re-Excision and Margin Findings

\begin{tabular}{|c|c|c|c|}
\hline $\begin{array}{c}\text { Re-Excision } \\
\text { Findings }\end{array}$ & $\begin{array}{c}\text { Final } \\
\text { Margin }\end{array}$ & $\begin{array}{c}\text { Patients } \\
\text { (No,) }\end{array}$ & $\begin{array}{c}\text { FFLR } \\
\text { (\%) }\end{array}$ \\
\hline \hline No residual & Negative & 168 & 96 \\
\hline Residual & All & 91 & 87 \\
\hline Residual & Negative & 58 & 92 \\
\hline Residual & Close, Positive, NA & 33 & 80 \\
\hline No re-excision & Negative & 115 & 94 \\
\hline
\end{tabular}

Extensive intraductal component (EIC) was the only factor identified in Cox regression analysis as a predictor of FFLR among patients with negative re-excision findings 
$(\mathrm{p}=.02)$. The FFLR was $96 \%$ in the absence of EIC and $81 \%$ in the presence of EIC.

Table 3. 10 Year Freedom from Local Recurrence Rates by Age and Tumor Bed Dose for Patients with Negative Re-Excision Findings

\begin{tabular}{|c|c|c|}
\hline \multirow{2}{*}{ Age } & \multicolumn{2}{|c|}{ Tumor Bed Dose } \\
\cline { 2 - 3 } & $\geq \mathbf{6 0} \mathbf{~ G y}$ & $<\mathbf{6 0} \mathbf{~ G y}$ \\
\hline \hline$<50$ years & $96 \%$ & $87 \%$ \\
\hline$\geq 50$ years & $94 \%$ & $100 \%$ \\
\hline
\end{tabular}

Table 4. Local Recurrence Rates for Margins by Re-excision Findings

\begin{tabular}{|c|c|c|c|}
\hline \multirow{2}{*}{ Series } & $\begin{array}{c}\text { Patients } \\
\text { (no.) }\end{array}$ & $\begin{array}{c}\text { Re-Excision } \\
\text { Finding }\end{array}$ & $\begin{array}{c}\text { Local } \\
\text { Recurrence (\%) }\end{array}$ \\
\hline \hline Park [13] & 101 & No residual & 8 \\
\hline \multirow{2}{*}{ Chism [9] } & 546 & No residual & 5 \\
\cline { 2 - 4 } & 299 & Residual & 9 \\
\hline Arthur [8] & 205 & No residual & 8 \\
\hline \multirow{2}{*}{ Pezner [12] } & 38 & No residual & 3 \\
\cline { 2 - 4 } & 29 & Residual & 12 \\
\hline \multirow{2}{*}{ Goldstein [14] } & 155 & No residual & 2 \\
\cline { 2 - 4 } & 286 & Residual & 11 \\
\hline \multirow{2}{*}{ Present } & 168 & No residual & 4 \\
\cline { 2 - 4 } & 91 & Residual & 13 \\
\hline
\end{tabular}

\section{DISCUSSION}

Breast-conserving surgery with whole breast radiation is an established treatment for early stage breast cancer. The final pathologic margin status of the lumpectomy specimen is generally the most important factor determining local recurrence rates in the majority of reports, although variation exists among institutions in terms of what constitutes an acceptable margin. The addition of a boost radiation dose has been demonstrated by large randomized trials to further reduce the risk of local recurrence in patients with negative margins, with the greatest absolute benefit occurring in patients under 50 years of age.

Few studies have reported results separately for patients undergoing re-excision according to the pathologic findings. Pezner et al. reported on 67 patients who underwent reexcision with negative margins (defined as no tumor at ink) and received whole breast radiation to $50 \mathrm{~Gy}$ without a boost [12]. Among the patients with no residual cancer in the specimen, the five-year local control rate was $97 \%$ compared to $88 \%$ when there was residual cancer present, and $96 \%$ in the 86 patients with initially clear margins who did not undergo re-excision ( $>0.1$ ). Chism et al. reported on 846 women who underwent re-excision prior to whole breast radiation with a boost [9]. The 10-year FFLR was 95\% for 546 patients with no residual tumor in the specimen and $91 \%$ for 299 patients with residual tumor $(\mathrm{p}=0.038)$, although 96 patients still had close, positive, or unknown margin status. Arthur et al. reported on 205 patients who had no residual tumor at the time of re-excision and were treated with $50 \mathrm{~Gy}$ whole breast radiation without a boost [8]. The 15-year local control rate was $92.4 \%$, and the risk of a true tumor bed recurrence was $2.4 \%$. Age $<50$ was a significant predictor for local recurrence in that series, with a FFLR of $87 \%$ as compared to $96 \%$ for those $\geq 50$ years $(\mathrm{p}=.0158$ ). However, 4 of 7 local recurrences in the younger age group were outside the tumor bed, and the authors concluded that the effect of a boost dose was therefore of uncertain benefit. Park et al. found an $8 \%$ risk of local recurrence at 8 years in 101 patients with no residual tumor at re-excision, which was not significantly different than the $7 \%$ risk of local recurrence for all patients with negative margins who received whole breast radiation followed by a boost [13]. Goldstein et al. reported on 583 patients with 607 invasive cancers treated with breast-conservation with a boost and found that the 12-year ipsilateral breast failure rate was $18 \%$ for 166 patients who did not undergo a re-excision, $11 \%$ for 286 patients with evidence of residual invasive or in situ carcinoma in the re-excision specimen, and $2 \%$ for 155 patients with no residual carcinoma in the specimen $(\mathrm{p}=.008)$.[14] A total radiation therapy dose $>60$ Gy was also associated with a lower 12-year ipsilateral breast failure rate (6\% compared to $21 \%$ and $31 \%$ for total doses of $51-60 \mathrm{~Gy}$ and $<51 \mathrm{~Gy}$, respectively, $\mathrm{p}=<.001$ ), although the final margin status in relation to total radiation dose is unclear.

Our results are in general supportive of previous findings. We found an excellent overall FFLR of $96 \%$ for patients with no residual tumor at re-excision. This did not appear significantly different, however, than the risk of local recurrence for patients with residual cancer in the re-excision specimen, as long as the final margins were negative. While some authors have suggested that patients with residual carcinoma in the re-excision specimen have a higher risk of local recurrence compared to those with no residual tumor $[12,14]$, the small number of patients in the current study and the definition of a negative margin $(>2 \mathrm{~mm})$ may have precluded any statistical significance in our findings. If we included patients with non-negative margins after reexcision, as Chism did in their comparison of patients with no residual and with residual tumor, we found a borderline statistically significant difference of $96 \%$ versus $87 \%$ FFLR, which is in agreement with their findings. Patients with residual tumor who do not achieve negative margins after reexcision appear to have a significantly higher risk of recurrence than those that have residual tumor and achieve negative margins.

The 10-year results of the EORTC randomized boost trial demonstrated a statistically significant benefit with the use of a boost to the lumpectomy cavity after whole breast radiotherapy [15]. While the relative risk was similar across all age groups, the absolute benefit was much greater in younger patients compared to older patients. The debate about whether to use a boost in an older patient population is sparked by the fact that the boost may not be cost-effective and may adversely affect cosmetic outcome. The EORTC trial, however, did not define subgroups of patients according to the presence or absence of residual disease in those patients who underwent re-excision. With the suggestion that a negative re-excision may offer a significant 
reduction in the overall tumor burden such that doses of 50 Gy might be adequate for local control, some authors have questioned the benefit of a boost in the setting of no residual tumor at re-excision [8]. Our results similarly found that among the entire cohort of patients with negative re-excision findings, there was no significant difference in the local recurrence rate between those who received a boost and those who did not. Whereas a large number of patients as in the EORTC trial would be necessary to show a statistically significant benefit with the boost treatment, these retrospective data are hypothesis-generating when thinking about individual patients receiving radiotherapy as a component of breast conservation therapy.

Although small patient numbers did not allow us to demonstrate statistical significance, patients $<50$ years of age despite having no residual tumor in the re-excision specimen tended to have an inferior rate of local control when a boost radiation dose was omitted, consistent with the results of Arthur et al.[8] Young patients $(<35-50)$ have been reported to have an increased risk of recurrence independent of margin status in many reports of breast conservation [16]. Some data indicate that young patients more often have closer margins of excision, as well as an increased incidence of DCIS, LVI, and other poor prognosis pathologic features [17]. Young age, along with EIC, was previously reported as a predictor of finding residual carcinoma at the time of reexcision in this patient population [10]. Even in patients with no residual tumor at re-excision, however, EIC was a significant predictor of local recurrence in the current series, in contrast to several series that have shown that EIC does not appear to be prognostic independent of margin status [18-21]. This may be a spurious observation, considering the small number of patients in this series with documented EIC and negative re-excision findings. Data from the larger EORTC boost trial demonstrate that patients $<40$ have a higher risk of local recurrence and a greater absolute benefit with a boost radiation dose [3, 15]; however, the study apparently allowed DCIS at the margins of excision in their definition of negative margins and, therefore, the interaction of young age and EIC is unclear. Finally, the small number of recurrences in our group of patients with negative reexcisions limits our ability to draw conclusions on the patterns of true versus elsewhere failures relative to the benefit of a tumor bed boost.

There are several limitations of this retrospective study. The institutional criteria and application of breast conservation undoubtedly varied over the 24 years of the study. In addition, wide variation in radiation and chemotherapy practices occurred over this time, and pathologic practices changed as well. Although we attempted to standardize the definition of various pathologic features and review available material at Stanford, not all material was reviewed by a single pathologist. In addition, the small number of patients in various subgroups limits the number of analyses that can be performed with any expectation of statistical significance. As is inherent in a retrospective study, there is patient and physician selection bias in terms of how patients were treated with regards to receiving a boost radiation dose. Due to the limitations of the retrospective nature of this study, it will be important to address this question in a prospective fashion before making any definitive conclusions or changes in current clinical practice.

In conclusion, the presence or absence of residual disease in the re-excision specimen did not predict for local recurrence as long as the final margins were $>$ or $=$ to $2 \mathrm{~mm}$. Patients with negative pathologic findings at re-excision did not appear to benefit significantly from the addition of a boost to the lumpectomy cavity after whole breast radiotherapy. There was a trend toward better local control, however, in younger patients treated with a boost even in the setting of a negative re-excision, consistent with the results of the EORTC trial.

\section{CONFLICT OF INTEREST}

The authors have no conflicts of interest to report.

\section{ACKNOWLEDGEMENT}

Approval for this work was obtained by the Institutional Review Board.

\section{REFERENCES}

[1] Early Breast Cancer Trialists' Collaborative Group. Effects of radiotherapy and surgery in early breast cancer. An overview of the randomized trials. N Engl J Med 1995; 333(22): 1444-55.

[2] Clarke M, Collins R, Darby S, et al. Effects of radiotherapy and of differences in the extent of surgery for early breast cancer on local recurrence and 15-year survival: an overview of the randomised trials. Lancet 2005; 366(9503): 2087-106.

[3] Bartelink H, Horiot JC, Poortmans P, et al. Recurrence rates after treatment of breast cancer with standard radiotherapy with or without additional radiation. N Engl J Med 2001; 345(19): 137887.

[4] Polgar C, Fodor J, Orosz Z, et al. Electron and high-dose-rate brachytherapy boost in the conservative treatment of stage I-II breast cancer first results of the randomized Budapest boost trial. Strahlenther Onkol 2002; 178(11): 615-23.

[5] Romestaing P, Lehingue Y, Carrie C, et al. Role of a 10-Gy boost in the conservative treatment of early breast cancer: results of a randomized clinical trial in Lyon, France. J Clin Oncol 1997; 15(3): 963-8.

[6] Hayman JA, Hillner BE, Harris JR, Pierce LJ, Weeks JC. Costeffectiveness of adding an electron-beam boost to tangential radiation therapy in patients with negative margins after conservative surgery for early-stage breast cancer. J Clin Oncol 2000; 18(2): 287-95.

[7] Vrieling C, Collette L, Fourquet A, et al. The influence of patient, tumor and treatment factors on the cosmetic results after breastconserving therapy in the EORTC 'boost vs no boost' trial. EORTC Radiotherapy and Breast Cancer Cooperative Groups. Radiother Oncol 2000 ; 55(3): 219-32.

[8] Arthur DW, Cuttino LW, Neuschatz AC, et al. Tumor bed boost omission after negative re-excision in breast-conservation treatment. Ann Surg Oncol 2006; 13(6): 794-801.

[9] Chism DB, Freedman GM, Li T, Anderson PR. Re-excision of margins before breast radiation-diagnostic or therapeutic? IJROBP 2006; 65(5): 1416-21.

[10] Smitt MC, Nowels K, Carlson RW, Jeffrey SS. Predictors of reexcision findings and recurrence after breast conservation. IJROBP 2003; 57(4): 979-85.

[11] Smitt MC, Nowels KW, Zdeblick MJ, et al. The importance of the lumpectomy surgical margin status in long-term results of breast conservation. Cancer 1995; 76(2): 259-67.

[12] Pezner RD, Wagman LD, Ben-Ezra J, Odom-Maryon T. Breast conservation therapy: local tumor control in patients with pathologically clear margins who receive 5000 cGy breast irradiation without local boost. Breast Cancer Res Treat 1994; 32(3): 261-7.

[13] Park CC, Mitsumori M, Nixon A, et al. Outcome at 8 years after breast-conserving surgery and radiation therapy for invasive breast cancer: influence of margin status and systemic therapy on local recurrence. J Clin Oncol 2000; 18(8): 1668-75. 
[14] Goldstein NS, Kestin L, Vicini F. Factors associated with ipsilateral breast failure and distant metastases in patients with invasive breast carcinoma treated with breast-conserving therapy. A clinicopathologic study of 607 neoplasms from 583 patients. Am J Clin Pathol 2003; 120(4): 500-27.

[15] Bartelink H, Horiot JC, Poortmans PM, et al. Impact of a higher radiation dose on local control and survival in breast-conserving therapy of early breast cancer: 10-year results of the randomized boost versus no boost EORTC 22881-10882 trial. J Clin Oncol 2007; 25(22): 3259-65.

[16] Zhou P, Recht A. Young age and outcome for women with earlystage invasive breast carcinoma. Cancer 2004; 101(6): 1264-74.

[17] Vrieling C, Collette L, Fourquet A, et al. Can patient-, treatmentand pathology-related characteristics explain the high local recurrence rate following breast-conserving therapy in young patients? Eur J Cancer 2003; 39(7): 932-44.
[18] Gage I, Schnitt SJ, Nixon AJ, et al. Pathologic margin involvement and the risk of recurrence in patients treated with breast-conserving therapy. Cancer 1996; 78(9): 1921-8.

[19] Burke MF, Allison R, Tripcony L. Conservative therapy of breast cancer in Queensland. Int J Radiat Oncol Biol Phys 1995; 31(2): 295-303.

[20] Schnitt SJ, Abner A, Gelman R, et al. The relationship between microscopic margins of resection and the risk of local recurrence in patients with breast cancer treated with breast-conserving surgery and radiation therapy. Cancer 1994; 74(6): 1746-51.

[21] Solin LJ, Fowble BL, Schultz DJ, Goodman RL. The significance of the pathology margins of the tumor excision on the outcome of patients treated with definitive irradiation for early stage breast cancer. Int J Radiat Oncol Biol Phys1991; 21(2): 279-87.

Received: August 30, 2011

Revised: September 12, 2011

Accepted: September 15, 2011

(C) Smitt and Horst; Licensee Bentham Open.

This is an open access article licensed under the terms of the Creative Commons Attribution Non-Commercial License (http://creativecommons.org/licenses/by-nc/ 3.0/) which permits unrestricted, non-commercial use, distribution and reproduction in any medium, provided the work is properly cited. 